${ }^{1}$ Centro de Excelencia en Medicina Traslacional, CEMTBIOREN, Universidad de La Frontera. Temuco, Chile. ${ }^{2}$ Centro de Investigación en Epidemiología Cardiovascular y Nutricional, EPICYN, Universidad de La Frontera. Temuco, Chile. ${ }^{3}$ Departamento de Medicina Interna, Universidad de La Frontera. Temuco, Chile. ${ }^{4}$ Departamento de Educación Física, Deportes y Recreación, Universidad de La Frontera. Temuco, Chile.

${ }^{5}$ Departamento de Enfermería, Universidad de La Frontera. Temuco, Chile.

${ }^{6}$ Departamento de Ciencias Básicas, Universidad de La Frontera. Temuco, Chile. ${ }^{a}$ Kinesiólogo; PhD en Estudios del Movimiento Humano

bBecado pediatría, Facultad de Medicina, Universidad de La Frontera, Temuco, Chile. 'Enfermero; $\mathrm{Mg}^{\circ}$ en Ciencias Médicas.

dKinesiólogo; Mg en Educación Física.

eBioquímico; $\mathrm{PhD}^{\oplus}$ en Biología Celular y Molecular Aplicada. fEstudiante de Tecnología Médica.

${ }^{9}$ Tecnólogo Médico; PhD en Farmacia área Análisis Clínico.

Trabajo financiado por Fondo de Investigación UNETE (Proyecto \# UNT15-004) del Convenio de Desempeño Regional, FRO 1301,

Universidad de La Frontera. Los autores declaran no tener conflictos de interés.

Recibido el 24 de mayo de 2018, aceptado el 11 de octubre de 2018.

Correspondencia a: Alvaro Cerda, PhD Departamento de Ciencias Básicas Universidad de La Frontera Centro de Excelencia en Medicina Traslacional.

Av. Alemania 0458 Temuco, Chile. alvaro.cerda@ufrontera.cl

\section{Alta prevalencia de dislipidemias y riesgo aterogénico en una población infanto-juvenil}

\author{
JORGE SAPUNAR ${ }^{1,2,3}$, NICOLÁS AGUILAR-FARÍAS ${ }^{2,4, a}$, \\ JUAN NAVARRO ${ }^{\mathrm{b}}$, GUSTAVO ARANEDA ${ }^{1,5, \mathrm{c}}$, \\ DAMIAN CHANDÍA-POBLETE ${ }^{4, d}$, VÍCTOR MANRÍQUEZ ${ }^{1, \mathrm{e}}$, \\ ROBERTO BRITO $^{1, \mathrm{f}}$, ÁLVARO CERDA ${ }^{1,2,6, \mathrm{~g}}$
}

\section{High prevalence of dyslipidemia and high atherogenic index of plasma in children and adolescents}

Background: Dyslipidemias in childhood increase the risk of cardiovascular events in adult life. Aim: To evaluate the prevalence of dyslipidemia and risk of atherogenicity based in the atherogenic index of plasma (AIP) in a sample of school children and adolescents. Material and Methods: Cross-sectional study of 208 children aged $10.4 \pm 1.0$ years (107 women). Demographic data were obtained, and a clinical evaluation was conducted, including pubertal development according to Tanner and anthropometric parameters. A fasting blood sample was obtained to measure total cholesterol (CT), HDL cholesterol (cHDL) and triglycerides (TG), glucose and insulin. LDL cholesterol (cLDL), Non-HDL cholesterol and the indices CT/cHDL, $c L D L / c H D L$ and AIP (log[TG/cHDL]) were calculated. Risk categories according to AIP for the pediatric population were also determined (low: AIP < 0.11, intermediate: AIP 0.11-0.21, high: AIP > 0.21). Results: Thirty eight percent of participants had dyslipidemia, without differences by gender and pubertal development. The frequency of dyslipidemia was significantly higher in children with obesity $(54 \%, p<0.01)$ and a waist circumference over percentile $90(61 \% ; p<0.01)$. The later conditions had also higher CT/cHDL, cLDL/cHDL and AIP. According to AIP, 54\% of children had a high atherogenicity risk along with alterations in anthropometric parameters and insulin resistance. All anthropometric and insulin resistance parameters were significantly correlated with the AIP. Conclusions: There is a high prevalence of dyslipidemia in the studied population, which is associated with an increased cardiometabolic risk. The indices of atherogenicity and particularly AIP are correlated with nutritional status, abdominal obesity and parameters of insulin resistance.

(Rev Med Chile 2018; 146: 1112-1122)

Key words: Cardiovascular Diseases; Child; Dyslipidemias; Risk Factors. 
$\mathrm{E}$ n Chile, las enfermedades cardiovasculares (ECV) constituyen la primera causa de mortalidad, dando cuenta de $27 \%$ de las defunciones anuales ${ }^{1}$. En la Encuesta Nacional de Salud (ENS) 2016-2017, 3,3\% de la muestra reconoció haber tenido un infarto agudo al miocardio y $2,6 \%$ un evento cerebrovascular, lo que representa un incremento significativo respecto a lo reportado en ENS 2010². El factor de riesgo de ECV más estudiado es la dislipidemia y su mecanismo patogénico es la ateroesclerosis, proceso que comienza en la niñez y progresa en forma gradual y silenciosa hasta expresarse en la vida adulta ${ }^{3}$.

Se ha reportado correlación positiva entre factores de riesgo cardiovascular presentes en la infancia y lesiones vasculares preclínicas, como mayor espesor de la íntima en arterias carótida y femoral en la vida adulta ${ }^{4,5}$. Dos grandes estudios de cohortes prospectivos encontraron que la concentración sérica de colesterol en la infancia permite predecir el valor de este parámetro en la vida adulta ${ }^{6,7}$. Por lo tanto, el análisis del perfil lipídico en población infanto-juvenil permitiría identificar e intervenir tempranamente sujetos en riesgo de desarrollar ECV en la vida adulta.

La prevalencia de dislipidemias en población infanto-juvenil es sorprendentemente alta. Un estudio de corte transversal con datos obtenidos del National Health and Nutrition Examination Survey (NHANES) entre los años 1999 y 2006 reportó que 20,6\% de los adolescentes de 12 a 19 años presentaban, a lo menos, un parámetro lipídico alterado ${ }^{8}$. Hallazgos similares se han reportado en Europa ${ }^{9,10}$, Asia ${ }^{11,12}$ y África ${ }^{13}$. En Latinoamérica, la prevalencia de dislipidemia infanto-juvenil alcanza valores tan alarmantes como $48,8 \%{ }^{14}$ y $62,1 \%{ }^{15}$. En Chile, Barja y colaboradores ${ }^{16}$ encontraron que $32 \%$ de una muestra de escolares de la ciudad de Santiago presentaba alguna forma clínica de dislipidemia.

Entre los factores asociados con mayor prevalencia de dislipidemia en población infanto-juvenil destacan la obesidad, el síndrome metabólico y determinantes de estos como la inactividad física ${ }^{16-17}$. El estatus socioeconómico y la ruralidad también están asociados con la presencia de dislipidemia y otros factores de riesgo cardiovascular ${ }^{18-20}$. En la ENS 2010, La Araucanía, en relación a otras regiones de Chile, presentó una alta prevalencia de trastornos nutricionales por exceso, diabetes mellitus, dislipidemia e hipertensión arterial ${ }^{21}$. Por otra parte, esta región tiene los peores indicadores socioeconómicos del país, una alta proporción de población rural y aborigen en algunas de sus comunas $^{22,23}$. La comuna de Carahue, ubicada en la costa de la provincia de Cautín en la Región de La Araucanía, es representativa de este perfil demográfico.

Por lo tanto, el propósito del presente estudio fue establecer la prevalencia de dislipidemia y de riesgo de aterogenicidad en escolares de la comuna de Carahue.

\section{Material y Métodos}

\section{Muestra de población}

Estudio de corte transversal que incluyó escolares de $4^{\circ}$ a $6^{\circ}$ año de enseñanza básica de la comuna de Carahue, Región de La Araucanía, entre noviembre 2015 y diciembre 2016. Los participantes del estudio fueron seleccionados mediante muestreo probabilístico aleatorio multinivel según tipo de establecimiento, ubicación geográfica y tamaño de la matrícula.

El protocolo de investigación fue aprobado por el Comité Ético Científico de la Universidad de La Frontera (Folio No 026/15). Los directores de cada establecimiento firmaron una autorización para evaluar su escuela. Luego, los apoderados o cuidadores de los escolares de cursos seleccionados firmaron un consentimiento informado, para proceder con el asentimiento de los escolares para realizar los procedimientos.

\section{Evaluación clínica, medidas antropométricas y estado nutricional}

Se recolectaron datos biodemográficos y se realizó evaluación clínica completa por médico pediatra, incluyendo desarrollo puberal y parámetros antropométricos.

Los participantes fueron clasificados de acuerdo a su desarrollo puberal en estadio de 1 a 5 según Tanner. Para el cálculo del índice de masa corporal (IMC) el peso y talla fueron medidos mediante balanza y estadiómetro. La circunferencia de cintura y de cadera fueron medidas con cinta métrica no extensible a nivel de ombligo y trocánteres, respectivamente, y con estas se calculó el índice cintura/cadera (ICC). Las presiones arteriales sistólica y diastólica (PAS y PAD) fueron medidas con esfigmomanómetro pediátrico, registrándose el promedio de 2 mediciones consecutivas. El es- 
tado nutricional se estableció mediante el criterio percentilar del Center for Disease Control (CDC) a través del cálculo del z-score del IMC normalizado por edad y sexo según recomendación de la Norma para la Evaluación Nutricional de Niños, Niñas y Adolescentes de 5 a 19 años de edad del Ministerio de Salud $2016^{24}$. Brevemente, individuos con IMC $\mathrm{z}$-score entre $-1 \mathrm{y}+1$ ( $\mathrm{p} 5$ a $\mathrm{p} 85$ ) fueron considerados eutróficos, aquellos con IMC z-score entre $+1 \mathrm{y}+2$ (p85 a p95) fueron considerados con sobrepeso y aquellos con valores mayores que +2 (> p95) fueron considerados obesos. Cuando se detectó una diferencia mayor a 1 año entre la edad cronológica y la edad biológica según su desarrollo puberal, se consideró esta última para el cálculo del IMC z-score. Se definió obesidad abdominal como el tener una circunferencia de cintura mayor que el percentil 90 de acuerdo a edad y sexo según la Norma para la Evaluación Nutricional de Niños, Niñas y Adolescentes de 5 a 19 años de edad del Ministerio de Salud 2016 24 . Los individuos con valores de PAS y PAD superiores al percentil 90 para su edad y sexo, según recomendación del National High Blood Pressure Education Program (NHBPEP), fueron considerados hipertensos ${ }^{25}$. Finalmente, se consignó la presencia de acantosis nigricans, el perímetro del brazo y cuello. La grasa corporal fue obtenida mediante impedanciometría (Tanita TBF300, Tanita Incorporation of America, Arlington Heights, IL, EUA).

\section{Parámetros bioquímicos, definición de dislipidemia e indices de aterogenicidad}

Se obtuvieron muestras de sangre en ayuno de 10 a 12 horas para determinar concentraciones séricas de glucosa, colesterol (CT) y triglicéridos (TG) a través de métodos enzimático-colorimétricos. La concentración de colesterol VLDL (cVLDL) fue calculada como la quinta parte de la concentración de triglicéridos (TG/5), la concentración de colesterol LDL (cLDL) fue calculada mediante la fórmula de Friedewald ${ }^{26}$ y la concentración de colesterol no-HDL (c-noHDL) fue calculada como la diferencia entre el colesterol total y colesterol HDL (cHDL). La insulinemia fue determinada por quimioluminiscencia. El análisis de los parámetros bioquímicos y hormonales fueron realizadas en equipos Roche-Cobas 311 y 411 (Roche Diagnostics, Basilea, Suiza).

La condición de dislipidemia se estableció por la presencia de al menos un parámetro del perfil lipídico alterado. Fueron utilizados los valores de corte propuestos por el National Cholesterol Education Program (NCEP) y American Academic of Pediatrics para población pediátrica ${ }^{27}$ :

- $\mathrm{CT} \geq 200 \mathrm{mg} / \mathrm{dL}$.

- $\quad c L D L \geq 130 \mathrm{mg} / \mathrm{dL}$.

- $\mathrm{cHDL}<40 \mathrm{mg} / \mathrm{dL}$.

- c-noHDL $\geq 145 \mathrm{mg} / \mathrm{dL}$.

- $\mathrm{TG} \geq 100 \mathrm{mg} / \mathrm{dL}$ entre $0-9$ años $\mathrm{y} \geq 130 \mathrm{mg} / \mathrm{dL}$ en mayores de 10 años.

También fueron calculados las razones CT/ cHDL, cLDL/cHDL y el índice de aterogenicidad del plasma (IAP), definido como log[TG/cHDL]. Las categorías de riesgo según IAP para población pediátrica fueron definidas como riesgo bajo (IAP $<0,11$ ), riesgo intermedio (IAP 0,11-0,21) y riesgo alto (IAP $>0,21$ ), de acuerdo a Vlavrik y colaboradores $^{28}$.

Para describir resistencia a la insulina (RI) se calcularon los índices HOMA-IR y QUICKI. La definición de RI se estableció de acuerdo a los criterios propuestos por Barja y colaboradores para población pediátrica chilena ${ }^{29}$.

\section{Análisis estadístico}

Se realizó un análisis descriptivo de los datos. La prueba de Kolmogorov-Smirnov fue usada para evaluar normalidad de variables continuas. Comparaciones entre grupos se realizaron usando pruebas de chi-cuadrado para variables categóricas y de t o ANOVA seguido de Tukey para variables continuas con distribución normal. Variables con distribución no paramétrica fueron comparadas mediante prueba de Mann-Withney o Kruskal-Wallis, seguido de método de Dunn. Un análisis de regresión logística utilizando modelo paso a paso de selección de variables fue usado para evaluar la contribución de variables clínicas y antropométricas para el riesgo de dislipidemias. Para evaluar la asociación entre variables continuas se realizó un análisis de correlación lineal y cálculo del coeficiente de correlación de Pearson. Se consideró una significancia de $5 \%$ para los análisis estadísticos.

\section{Resultados}

Fueron evaluados 208 escolares (101 varones y 107 mujeres) con edad promedio de 10,4 $\pm 1,0$ años. En la Tabla 1 se resumen las variables clínicas 
y antropométricas de la muestra de acuerdo al sexo. El $80 \%$ de los varones y $61 \%$ de las mujeres presentaron trastornos nutricionales por exceso. En el caso de obesidad, los niños presentaron prevalencias mayores que las niñas ( $40 \%$ vs $27 \%$, respectivamente; $\mathrm{p}=0,029)$. También fueron observados valores más altos de IMC z-score y perímetro cervical, así como menor porcentaje de grasa corporal en los varones en comparación a las niñas $(\mathrm{p}<0,05)$.

En la Tabla 2 se presentan los parámetros bioquímicos de la población estudiada de acuerdo

Tabla 1. Datos demográficos, clínicos y antropométricos de participantes del estudio en el grupo total y según sexo

\begin{tabular}{|c|c|c|c|c|}
\hline Variable & Grupo total (208) & Masculino (101) & Femenino (107) & Valor $p$ \\
\hline Edad, años & $10,4 \pm 1,0$ & $10,31 \pm 1,001$ & $10,41 \pm 1,05$ & 0,478 \\
\hline Estadio Tanner (1-2/3-5), \% & $66 / 34$ & $85 / 15$ & $49 / 51$ & $<0,001$ \\
\hline PAS, $\mathrm{mmHg}$ & $109,6 \pm 18,6$ & $109,89 \pm 17,96$ & $109,35 \pm 19,44$ & 0,857 \\
\hline $\mathrm{PAD}, \mathrm{mmHg}$ & $61,5 \pm 16,7$ & $60,16 \pm 18,23$ & $62,95 \pm 14,91$ & 0,301 \\
\hline Peso, kg & $46,56 \pm 10,90$ & $46,23 \pm 10,73$ & $46,87 \pm 11,11$ & 0,674 \\
\hline IMC z-score & $1,50 \pm 0,97$ & $1,69 \pm 0,93$ & $1,32 \pm 0,97$ & 0,005 \\
\hline $\begin{array}{l}\text { Estado nutricional } \\
\text { Eutrófico } \\
\text { Sobrepeso } \\
\text { Obeso }\end{array}$ & $\begin{array}{l}29 \%(60) \\
38 \%(79) \\
33 \%(69)\end{array}$ & $\begin{array}{l}20 \%(21) \\
40 \%(40) \\
40 \%(40)\end{array}$ & $\begin{array}{l}36 \%(39) \\
36 \%(39) \\
27 \%(29)\end{array}$ & 0,029 \\
\hline$C A, c m$ & $71,9 \pm 9,7$ & $72,6 \pm 9,6$ & $71,1 \pm 9,4$ & 0,260 \\
\hline Obesidad abdominal & $26 \%(54)$ & $27 \%(27)$ & $26 \%(28)$ & 0,897 \\
\hline Circunferencia brazo, cm & $22,6 \pm 2,8$ & $22,8 \pm 2,8$ & $22,4 \pm 2,8$ & 0,346 \\
\hline Perímetro cervical, cm & $30,9 \pm 2,6$ & $31,4 \pm 2,5$ & $30,4 \pm 2,6$ & 0,007 \\
\hline Grasa corporal, \% & $28,4 \pm 8,7$ & $26,2 \pm 8,6$ & $30,5 \pm 8,4$ & 0,001 \\
\hline
\end{tabular}

Número de individuos en paréntesis. Variables categóricas son presentadas como porcentaje y comparadas por prueba de $\chi^{2}$. Variables continuas son presentadas como media \pm desvío estándar y fueron comparadas por prueba de t o Mann-Whitney para variables con distribución paramétrica o no paramétricas, respectivamente. PAS: Presión arterial sistólica, PAD: Presión arterial diastólica, IMC: Índice de masa corporal, CA: Circunferencia abdominal.

Tabla 2. Parámetros bioquímicos y hormonales de participantes del estudio en el grupo total y de acuerdo al sexo

\begin{tabular}{|lcccc|}
\hline Variable & Grupo total (208) & Masculino (101) & Femenino (107) & Valor p \\
\hline Glucosa, $\mathrm{mg} / \mathrm{dL}$ & $85,3 \pm 7,3$ & $86,3 \pm 6,3$ & $84,3 \pm 8,0$ & 0,053 \\
\hline Insulina, $\mu \mathrm{U} / \mathrm{mL}$ & $14,1 \pm 9,4$ & $13,0 \pm 10,4$ & $15,1 \pm 8,2$ & 0,097 \\
\hline HOMA-IR & $3,00 \pm 2,18$ & $2,82 \pm 2,47$ & $3,18 \pm 1,84$ & 0,244 \\
\hline Quicki & $0,337 \pm 0,033$ & $0,343 \pm 0,037$ & $0,330 \pm 0,027$ & 0,006 \\
\hline Colesterol total, mg/dL & $154,0 \pm 28,9$ & $153,9 \pm 28,2$ & $154,0 \pm 29,6$ & 0,979 \\
\hline Triglicéridos, mg/dL & $99,4 \pm 68,2$ & $104,1 \pm 86,5$ & $94,8 \pm 68,2$ & 0,336 \\
\hline Colesterol LDL, mg/dL & $84,0 \pm 23,9$ & $82,4 \pm 23,8$ & $85,6 \pm 23,8$ & 0,341 \\
\hline Colesterol HDL, mg/dL & $50,5 \pm 13,4$ & $51,6 \pm 13,9$ & $49,5 \pm 12,9$ & 0,267 \\
\hline Colesterol no-HDL, mg/dL & $103,5 \pm 28,0$ & $102,4 \pm 28,8$ & $104,6 \pm 27,3$ & 0,577 \\
\hline Colesterol VLDL, mg/dL & $19,9 \pm 13,7$ & $19,0 \pm 8,7$ & $20,9 \pm 17,4$ & 0,319 \\
\hline
\end{tabular}

Número de individuos en paréntesis. Variables categóricas son presentadas como porcentaje y comparadas por prueba de $\chi^{2}$. Variables continuas son presentadas como media \pm desvío estándar y fueron comparadas por prueba de t o Mann-Whitney para variables con distribución paramétrica o no paramétricas, respectivamente. LDL: lipoproteína de baja densidad, HDL: lipoproteína de alta densidad, VLDL: lipoproteína de muy baja densidad, HOMA-IR: Homeostatic model assessment-insulin resistance, Quicki: Quantitative Insulin Sensitivity Check Index. 
al sexo. No se observaron diferencias según sexo, excepto para el índice Quicki que mostró valores disminuidos en el sexo femenino $(\mathrm{p}=0,006)$.

El 38\% de los escolares presentó dislipidemia, sin diferencias por género y estado del desarrollo puberal. La prevalencia de dislipidemia fue significativamente mayor en sujetos con obesidad ( $54 \%$ vs $34 \%$ en sobrepeso y $25 \%$ en normopeso; $\mathrm{p}=0,002)$ y con obesidad abdominal $(61 \%$; $\mathrm{p}=0,001)$ (Tabla 3$)$.

En la Tabla 4 se presenta la prevalencia de alteraciones en parámetros del perfil lipídico según estado nutricional y presencia de obesidad abdominal. Las alteraciones del perfil lipídico asociadas con el estado nutricional y la presencia de obesidad abdominal fueron la elevación de triglicéridos y la disminución de cHDL, mientras que el c-noHDL elevado fue también más prevalente en sujetos con obesidad abdominal. La hiperlipidemia mixta y dislipidemia aterogénica fueron también más prevalentes en sujetos obesos y con obesidad abdominal. Un análisis de regresión múltiple paso a paso mostró que la obesidad abdominal contribuye para dislipidemia y todas sus formas más frecuentes (Tabla 5), siendo que perímetro cervical también se asocia a dislipidemia y la edad con triglicéridos elevado y cHDL bajo.

El valor de los índices CT/cHDL, cLDL/cHDL
Tabla 3. Prevalencia de dislipidemia en la población de estudio de acuerdo a sexo, desarrollo puberal, estado nutricional y obesidad abdominal

\begin{tabular}{|c|c|}
\hline & Dislipidemia \\
\hline $\begin{array}{ll}\text { Sexo } & \\
\text { Masculino } & (n=101) \\
\text { Femenino } & (n=107) \\
& \quad \chi^{2}=0,489 ; G L\end{array}$ & $\begin{array}{c}36 \%(36) \\
40 \%(42) \\
=1 ; p=0,485\end{array}$ \\
\hline 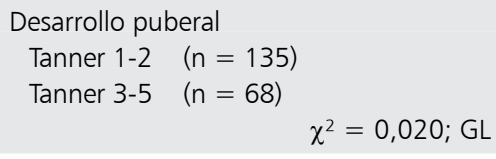 & $\begin{array}{c}38 \%(51) \\
37 \%(25) \\
=1 ; p=0,888\end{array}$ \\
\hline $\begin{array}{ll}\text { Estado nutricional } \\
\begin{array}{ll}\text { Normopeso } & (\mathrm{n}=57) \\
\text { Sobrepeso } & (\mathrm{n}=79) \\
\text { Obeso } & (\mathrm{n}=68) \\
& \quad \chi^{2}=12,646 ; \mathrm{GL}\end{array}\end{array}$ & $\begin{array}{c}25 \%(14) \\
34 \%(27) \\
54 \%(37) \\
=2 ; p=0,002\end{array}$ \\
\hline $\begin{array}{l}\text { Obesidad abdominal } \\
\text { Circunferencia cintura }<\text { p90 }(\mathrm{n}=150) \\
\text { Circunferencia cintura }>\text { p90 }(\mathrm{n}=54) \\
\qquad \chi^{2}=16,274 ; \mathrm{GL}\end{array}$ & $\begin{array}{c}30 \%(45) \\
61 \%(33) \\
=1 ; p<0,001\end{array}$ \\
\hline
\end{tabular}

Datos de son presentados en porcentaje de individuos con dislipidemia en cada uno de los subgrupos de análisis. Comparaciones de la frecuencia de dislipidemia de acuerdo al desarrollo puberal, estado nutricional y obesidad abdominal fueron realizadas mediante un análisis de chi-cuadrado $\left(\chi^{2}\right)$. El número de individuos se encuentra entre paréntesis. GL: Grados de libertad, p90: percentil 90.

\section{Tabla 4. Prevalencia de dislipidemia de acuerdo al parámetro del perfil lipídico alterado según estado} nutricional y obesidad abdominal

\begin{tabular}{|c|c|c|c|c|c|c|c|c|}
\hline \multirow{3}{*}{$\begin{array}{l}\text { Parámetro alterado } \\
\text { Colesterol total elevado }\end{array}$} & \multirow{3}{*}{$\begin{array}{c}\begin{array}{c}\text { Grupo } \\
\text { total }\end{array} \\
7 \%(14)\end{array}$} & \multirow{3}{*}{$\begin{array}{c}\text { Eutrófico } \\
5 \%(3)\end{array}$} & \multicolumn{2}{|c|}{$\begin{array}{l}\text { Estado nutricional } \\
\text { Sobrepeso Obeso }\end{array}$} & \multirow[b]{2}{*}{ Valor $\mathbf{p}$} & \multicolumn{3}{|c|}{ Obesidad abdominal } \\
\hline & & & Sobrepeso & Obeso & & CC $<$ p90 & CC $>$ p90 & Valor $\mathbf{p}$ \\
\hline & & & $8 \%(6)$ & $8 \% \quad(5)$ & 0,845 & $5 \%(8)$ & $11 \% \quad(6)$ & 0,169 \\
\hline Triglicéridos elevado & $21 \%(43)$ & $9 \%(5)$ & $15 \%(12)$ & $38 \%(26)$ & 0,001 & $15 \%(22)$ & $39 \%(21)$ & 0,001 \\
\hline Colesterol LDL elevado & $5 \%(11)$ & $4 \%(2)$ & $6 \%(5)$ & $6 \%$ & 0,737 & $4 \%(6)$ & $9 \% \quad(5)$ & 0,142 \\
\hline Colesterol HDL bajo & $23 \%(47)$ & $12 \%(7)$ & $15 \%(12)$ & $41 \%(28)$ & 0,001 & $15 \%(22)$ & $46 \%(25)$ & 0,001 \\
\hline Colesterol no-HDL elevado & $8 \%(15)$ & $4 \%(2)$ & $8 \% \quad(6)$ & $11 \% \quad(7)$ & 0,318 & $5 \%(7)$ & $15 \%$ & 0,021 \\
\hline Hiperlipidemia mixta & $3 \% \quad(6)$ & $2 \%(1)$ & $0 \% \quad(0)$ & $8 \% \quad(5)$ & 0,015 & $1 \%$ & $9 \% \quad(5)$ & 0,001 \\
\hline Dislipidemia aterogénica & $11 \%(21)$ & $2 \%(1)$ & $4 \% \quad(3)$ & $25 \%(17)$ & $<0,001$ & $5 \%(7)$ & $26 \%(14)$ & $<0,001$ \\
\hline
\end{tabular}

Datos de son presentados en porcentaje de individuos con parámetros del perfil lipídico alterado en el grupo total y en cada subgrupo de análisis. Comparaciones de la frecuencia de alteraciones del perfil lipídico de acuerdo al estado nutricional y obesidad abdominal fueron realizadas mediante análisis de chi-cuadrado $\left(\chi^{2}\right)$. El número de individuos se encuentra entre paréntesis. Valores de corte para parámetros alterados del perfil lipídico fueron determinados según recomendación del National Cholesterol Education Program (NCEP) y American Academic of Pediatrics para población pediátrica. Individuos con valores elevados de colesterol total y/o LDL y de triglicéridos fueron considerados como portadores de hiperlipidemia mixta, mientras que dislipidemia aterogénica fue definida como tener triglicéridos elevados y colesterol HDL bajo. Obesidad abdominal fue definida para cada individuo como circunferencia de cintura (CC) con valor superior al percentil 90 (CC >p90) de acuerdo a su edad y sexo según normas para evaluación nutricional de niños y niñas del Minsal. LDL: lipoproteína de baja densidad, HDL: lipoproteína de alta densidad. 
Tabla 5. Análisis de regresión logística para las formas más frecuentes de dislipidemia

\begin{tabular}{|c|c|c|c|c|c|c|c|c|}
\hline \multirow[t]{3}{*}{ Parámetro } & \multicolumn{8}{|c|}{ Forma de dislipidemia } \\
\hline & \multicolumn{2}{|c|}{ Dislipidemia } & \multicolumn{2}{|c|}{$\begin{array}{l}\text { Triglicéridos } \\
\text { elevado }\end{array}$} & \multicolumn{2}{|c|}{ Colesterol HDL bajo } & \multicolumn{2}{|c|}{$\begin{array}{l}\text { Dislipidemia } \\
\text { aterogénica }\end{array}$} \\
\hline & $\begin{array}{c}\text { OR } \\
\text { (IC 95\%) }\end{array}$ & Valor $\mathbf{p}$ & $\begin{array}{c}\text { OR } \\
\text { (IC } 95 \%)\end{array}$ & Valor p & $\begin{array}{c}\text { OR } \\
\text { (IC 95\%) }\end{array}$ & Valor $\mathbf{p}$ & $\begin{array}{c}\text { OR } \\
\text { (IC 95\%) }\end{array}$ & Valor $\mathbf{p}$ \\
\hline Obesidad abdominal & $\begin{array}{c}2,29 \\
(1,12-5,14)\end{array}$ & 0,029 & $\begin{array}{c}3,75 \\
(1,81-7,74)\end{array}$ & $<0,001$ & $\begin{array}{c}5,42 \\
(2,63-11,17)\end{array}$ & $<0,001$ & $\begin{array}{c}7,15 \\
(2,70-18,91)\end{array}$ & $<0,001$ \\
\hline Perímetro cervical & $\begin{array}{c}1,17 \\
(1,02-1,36)\end{array}$ & 0,042 & - & - & - & - & - & - \\
\hline Edad & - & - & $\begin{array}{c}0,64 \\
(0,44-0,92)\end{array}$ & 0,015 & $\begin{array}{c}1,51 \\
(1,07-2,14)\end{array}$ & 0,019 & - & - \\
\hline
\end{tabular}

Variables fueron seleccionadas utilizando modelo paso a paso de selección de variables, incluyendo diferentes formas de dislipidemia como variable dependiente y principales parámetros clínicos y medidas antropométricas como variables explicativas. OR: Odds Ratio; IC 95\%: Intervalo de confianza de 95\%; HDL: lipoproteína de alta densidad.

e IAP no varió en relación al género ni al desarrollo puberal, pero sí por el estado nutricional y la presencia de obesidad abdominal $(\mathrm{p}<0,001)$ (Tabla 6).

El $54,3 \%$ de los sujetos presentaron riesgo elevado de aterogenicidad de acuerdo al IAP. En la Tabla 7 son presentados los parámetros antropométricos y de resistencia a la insulina de acuerdo a categoría de riesgo según valor de IAP. Todos los parámetros antropométricos, así como insulinemia e índices de resistencia a la insulina estuvieron aumentados en el grupo de mayor riesgo $(\mathrm{p}<0,001)$. De la misma forma, individuos con mayor riesgo aterogénico según el IAP mostraron

Tabla 6. Valores de diferentes índices de aterogenicidad según sexo, desarrollo puberal, estado nutricional y obesidad abdominal en la población de estudio

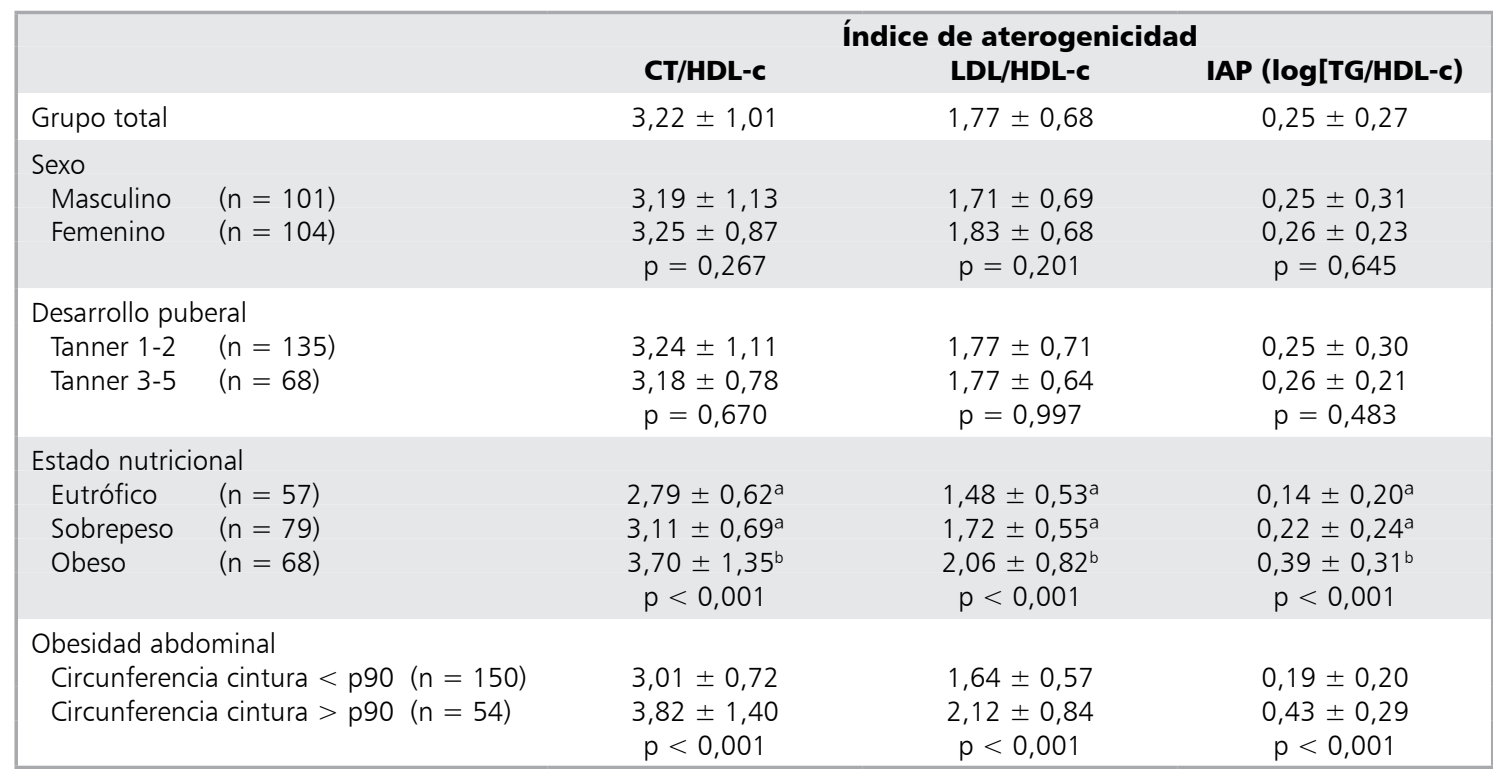

Datos son presentados como media \pm desviación estándar y fueron comparados por prueba de $t$ (Anova para estado nutricional) o Mann-Whitney (Kruskal-Wallis para estado nutricional) para variables con distribución paramétrica o no paramétricas, respectivamente. El número de individuos se encuentra entre paréntesis. Obesidad abdominal fue definida para cada individuo como circunferencia de cintura con valor superior al percentil 90 (CC > p90) de acuerdo a su edad y sexo según normas para evaluación nutricional de niños y niñas del Minsal. CT: Colesterol total, HDL-c: colesterol de la lipodroteina de alta densidad, TG: triglicéridos, IAP: Índice de aterogenicidad del plasma, p90: percentil 90. 
mayor prevalencia de obesidad, obesidad abdominal, RI y acantosis nigricans $(\mathrm{p}<0,001)$ (Figura 1$)$.

Como se puede observar en la Figura 2, todos los parámetros antropométricos se correlaciona- ran positivamente con el IAP. El mismo comportamiento fue observado para insulinemia y el HOMA-IR, mientras que el índice Quicki correlacionó negativamente con el riesgo de aterogenicidad.

Tabla 7. Medidas antropométricas e índices de resistencia a la insulina de acuerdo a categoría de riesgo según el índice de aterogenicidad del plasma (AIP)

\begin{tabular}{|lcccc|}
\hline & \multicolumn{3}{c|}{ Categoría de riesgo según IAP } \\
Parámetro & Bajo & Intermedio & Alto & Valor p \\
Número de individuos & 55 & 37 & 113 & \\
Parámetros antropométricos & & & & \\
$\quad$ Peso, Kg & $41,7 \pm 10,3^{\mathrm{a}}$ & $44,2 \pm 9,4^{\mathrm{a}}$ & $49,9 \pm 10,6^{\mathrm{b}}$ & $<0,001$ \\
IMC $z$-score & $1,03 \pm 0,99^{\mathrm{a}}$ & $1,41 \pm 0,86^{\mathrm{a}, \mathrm{b}}$ & $1,78 \pm 0,9^{\mathrm{b}}$ & $<0,001$ \\
CA, cm & $66,7 \pm 8,9^{\mathrm{a}}$ & $70,0 \pm 7,2^{\mathrm{a}}$ & $75,2 \pm 9,4^{\mathrm{b}}$ & $<0,001$ \\
Circunferencia brazo, cm & $21,3 \pm 2,6^{\mathrm{a}}$ & $22,0 \pm 2,7^{\mathrm{a}}$ & $23,5 \pm 1,1^{\mathrm{b}}$ & $<0,001$ \\
Perímetro cervical, cm & $23,6 \pm 2,6^{\mathrm{a}}$ & $30,5 \pm 2,0^{\mathrm{a}}$ & $31,7 \pm 2,4^{\mathrm{b}}$ & $<0,001$ \\
Grasa corporal, \% & $23,9 \pm 8,1^{\mathrm{a}}$ & $25,4 \pm 2,7^{\mathrm{a}}$ & $31,8 \pm 8,3^{\mathrm{b}}$ & $<0,001$ \\
Parámetros de resistencia a la insulina & & & & \\
$\quad$ Glucosa, mg/dL & $84,2 \pm 5,4$ & $86,2 \pm 6,3$ & $85,4 \pm 8,2$ & 0,389 \\
Insulina, $\mu \mathrm{U} / \mathrm{mL}$ & $10,1 \pm 6,0^{\mathrm{a}}$ & $14,7 \pm 9,7^{\mathrm{b}}$ & $15,8 \pm 10,1^{\mathrm{b}}$ & $<0,001$ \\
HOMA-IR & $2,12 \pm 1,24^{\mathrm{a}}$ & $3,14 \pm 2,05^{\mathrm{b}}$ & $3,38 \pm 2,46^{\mathrm{b}}$ & $<0,001$ \\
Quicki & $0,353 \pm 0,040^{\mathrm{a}}$ & $0,333 \pm 0,030^{\mathrm{b}}$ & $0,329 \pm 0,028^{\mathrm{b}}$ & $<0,001$ \\
\hline
\end{tabular}

Datos son presentados como media \pm desviación estándar y fueron comparados por ANOVA seguido de Tukey para variables con distribución paramétrica o usando Kruskall-Wallis seguido de método de comparación de Dunnett en el caso de variables no paramétricas. Las categorías de riesgo según el Índice de aterogenicidad del plasma (AIP) fueron definidas como de riesgo bajo (AIP $<0,11$ ), riesgo intermedio (AIP 0,11-0,21) o riesgo alto (AIP >0,21). IMC: Índice de masa corporal, CA: Circunferencia abdominal, HOMA-IR: Homeostatic model assessment-insulin resistance, Quicki: Quantitative Insulin Sensitivity Check Index.
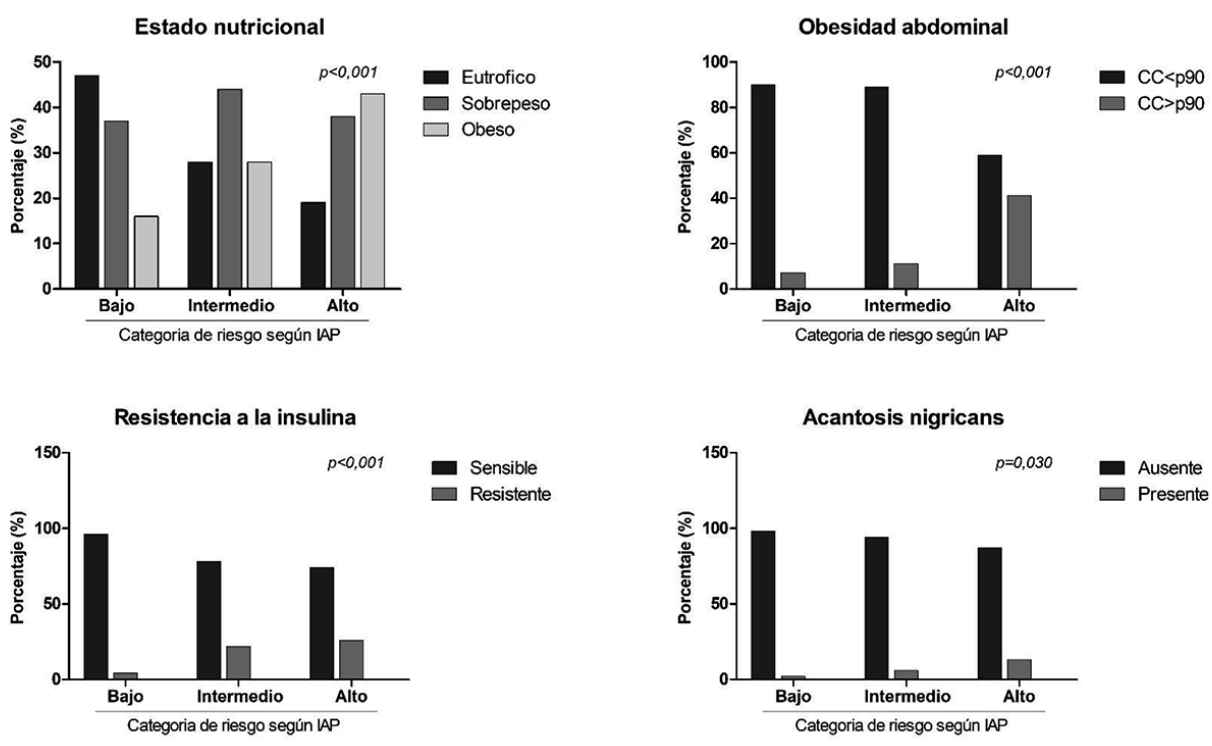

Figura 1. Factores de riesgo cardiometabólico de acuerdo a categoría de riesgo según el índice de aterogenicidad del plasma (AIP). Datos son presentados en porcentaje de individuos en cada grupo de acuerdo a categorías de riesgo según el AIP, las cuales fueron definidas como de riesgo bajo (AIP $<0,11$ ), riesgo intermedio (AIP $0,11-0,21)$ o riesgo alto (AIP $>0,21)$. Comparaciones de frecuencias fueron realizadas mediante análisis de chi-cuadrado $\left(\chi^{2}\right)$. CC < p90: Circunferencia de cintura menor que el $90^{\circ}$ percentil, CC > p90: Circunferencia de cintura mayor que el percentil 90 . 
A

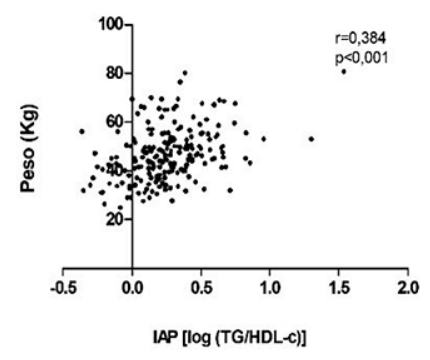

D

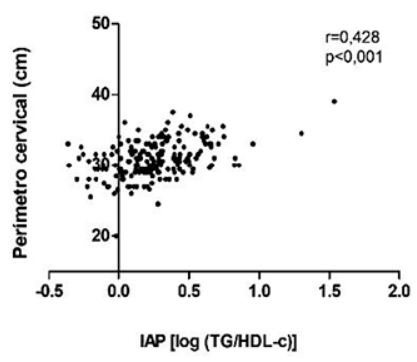

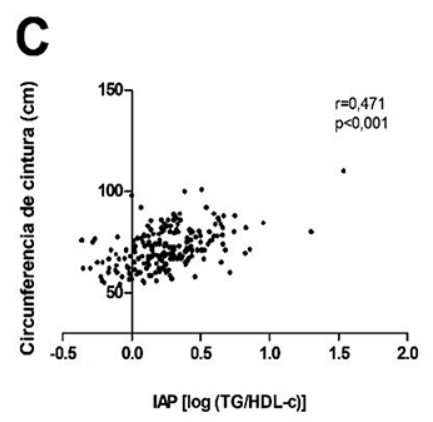

E

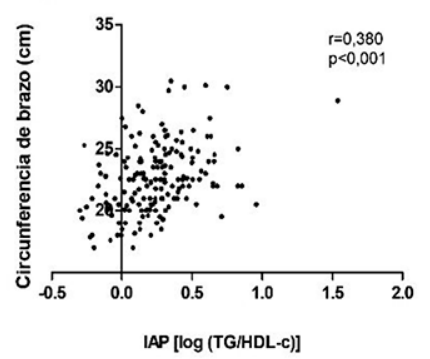

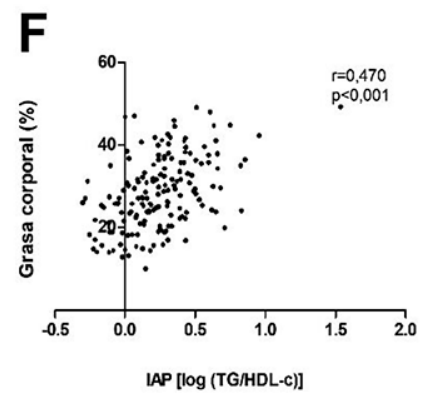

I

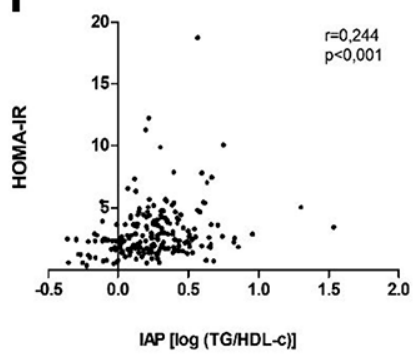

G
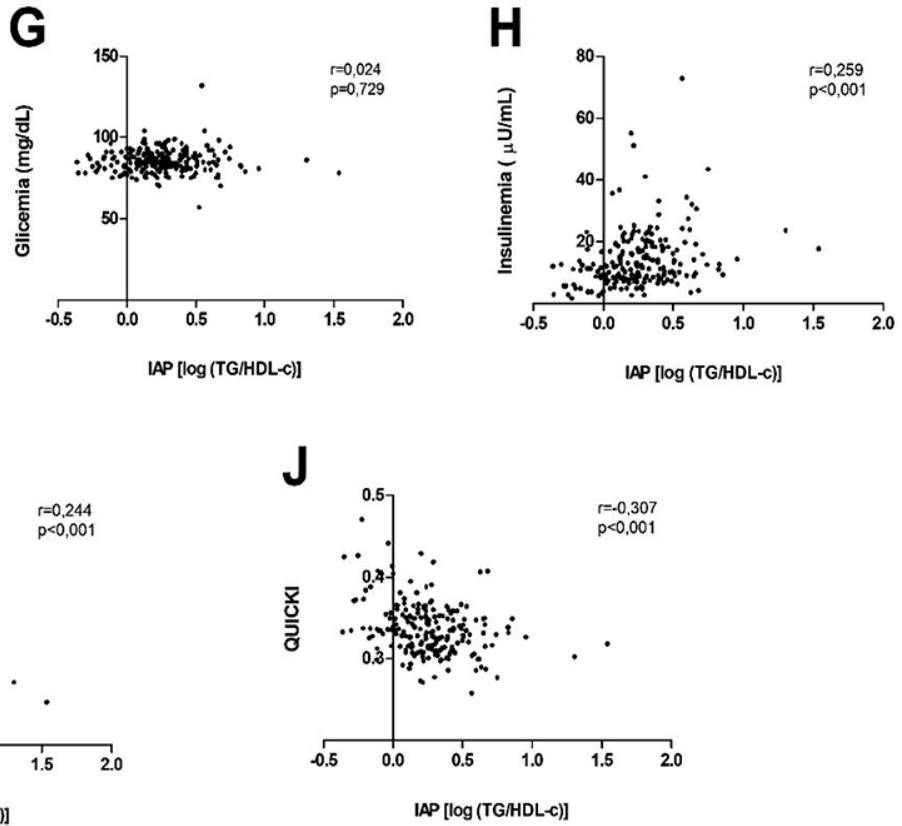

Figura 2. Correlacion de IAP con parámetros antropométricos y de resistencia a la insulina. Correlaciones del índice de aterogenicidad del plasma (AIP) con (A) peso corporal; (B) IMC z-score; (C) circunferencia de cintura; (D) perímetro cervical, (E) circunferencia de brazo, (F) grasa corporal, (G) glicemia, (H) insulinemia y (J) HOMA-IR y (I) QUICKI fueron evaluadas a través de Coeficiente de Correlación de Pearson (r). IMC: Índice de masa corporal, HOMA-IR: Homeostatic model assessment-insulin resistance; Quicki: Quantitative Insulin Sensitivity Check Index. 


\section{Discusión}

En nuestra muestra de escolares de la comuna de Carahue encontramos una prevalencia de $33,1 \%$ de obesidad, en tanto que en el estudio de Barja solo 15,3\% de la muestra de escolares de la comuna de Puente Alto, Región Metropolitana, estaban en esta categoría nutricional ${ }^{16}$. De acuerdo a la encuesta CASEN 2013, 41,91\% de los habitantes de la comuna de Carahue se encontraba en situación de pobreza por ingresos, en cambio en Puente Alto solo 14,6\%, lo que sugiere marcadas diferencias socioeconómicas en las características de población estudiada. Por otro lado, en el censo 2002, 29,02\% de la población de Carahue reconoció pertenecer a la etnia Mapuche, cifra que solo llegó a 2,99\% en Puente Alto ${ }^{30,31}$. Dichas diferencias en el nivel socioeconómico y en el origen étnico, así como el aumento sostenido de la obesidad infanto-juvenil en los últimos años, sugieren que las diferencias en relación a las características de la muestra estudiada podrían explicar la alta prevalencia de obesidad observada en nuestra población en relación al estudio previo. Un hallazgo de nuestro estudio fue la mayor proporción de individuos con indicadores nutricionales desfavorables en el sexo masculino, aspecto que lamentablemente no fue analizado en el estudio de Barja ni en otros realizados en Latinoamérica ${ }^{14-16}$.

El 38\% de los escolares tuvo algún tipo de alteración del perfil lipídico, proporción que fue significativamente mayor en sujetos con obesidad respecto de los grupos sobrepeso y normopeso, pero sin diferencias por género y estado del desarrollo puberal. En el estudio de Barja, la frecuencia de dislipidemia clínica fue $32 \%$, siendo también mayor en sujetos obesos ${ }^{16}$. En ambos estudios la disminución del cHDL y la elevación de TG fueron las alteraciones del perfil lipídico más frecuentes, particularmente en sujetos con obesidad.

El mayor riesgo cardiovascular lo confiere la presencia simultánea de cHDL bajo, cLDL y TG elevados, asociación denominada dislipidemia aterogénica ${ }^{32}$. En un esfuerzo por lograr un marcador de dislipidemia aterogénica se ha propuesto el IAP, el cual se ha mostrado más promisorio que la medición de otros parámetros lipídicos en la estimación del riesgo de $\mathrm{ECV}^{33}$, probablemente debido al hecho de que existe una fuerte correlación con el tamaño de partículas de lipoproteínas, como el aumento de LDL pequeñas y más densas asociado a un aumento de IAP. En nuestro estudio, 54,3\% de los escolares tuvo riesgo elevado de aterogenicidad de acuerdo al IAP, cifra que contrasta con 9,5\% comunicado por Vrablik y colaboradores para escolares de la República $\mathrm{Checa}^{28}$. En ambos estudios se demostró una fuerte asociación entre el valor de IAP y el estado nutricional, la diferencia es que la frecuencia de obesidad en escolares de Carahue fue 33,1\% y en la República Checa solo 6\%. Igualmente, llama la atención la correlación observada entre el IAP y medidas antropométricas, particularmente con la circunferencia de cintura y perímetro cervical, lo cual evidencia la importancia de una evaluación clínica exhaustiva que considere estos parámetros como potenciales indicadores de la presencia de dislipidemia. Sin embargo, se necesita explorar más profundamente este aspecto en otras poblaciones en el país e idealmente con un diseño prospectivo. Finalmente, pudimos establecer una asociación entre indicadores de resistencia a la insulina y riesgo de aterogenicidad. En su conjunto, los resultados obtenidos en torno al análisis de IAP en nuestra población sugieren que el uso de este índice puede ser útil para la caracterización del riesgo cardiometabólico en población infanto-juvenil, como propuesto recientemente en población turca, donde se concluyó que el IAP es superior al uso de otros índices de aterogenicidad y parámetros del perfil lipídico ${ }^{34}$.

En conclusión, escolares chilenos provenientes de una comuna con altos índices de pobreza y ruralidad mostraron un riesgo de aterogenicidad elevado basado en el uso del IAP, fuertemente vinculado a una alta prevalencia de obesidad. Estos hallazgos probablemente se explican por la asociación entre resistencia a la insulina y dislipidemia aterogénica. Además, se observó que medidas antropométricas simples, como la circunferencia de cintura, cuello y brazo tienen un potencial para ser evaluados como identificadores de riesgo de aterogenicidad elevado.

Agradecimientos: Agradecemos a todos los participantes y funcionarios de escuelas y Departamento de Educación Municipal de la comuna de Carahue que voluntariamente contribuyeron con esta investigación. El presente trabajo fue financiado por el Fondo de Investigación UNETE (\#UNT15-004) del Convenio de Desempeño Regional, FRO 1301, Universidad de La Frontera, 
Proyecto SOCHED No 2017-17 de la Sociedad Chilena de Endocrinología Diabetes y FONDECYT \#11150445.

\section{Referencias}

1. Disponible en: http://www.deis.cl/estadisticas-mortalidad/

2. Disponible en: http://web.minsal.cl/wp-content/ uploads/2017/11/ENS-2016-17_PRIMEROS-RESULTADOS.pdf

3. de Ferranti SD. Childhood cholesterol disorders: the iceberg base or nondisease? Med Clin North Am 2012; 96: 141-54.

4. Newman WP, Freedman DS, Voors AW, Gard PD, Srinvasan SR, Cresanta JL, et al. Relation of serum lipoprotein levels and systolic blood pressure to early atherosclerosis. The Bogalusa Heart Study. N Engl J Med 1986; 314: 138-44.

5. Davis PH, Dawson JD, Riley WA, Lauer RM. Carotid intimal-medial thickness is related to cardiovascular risk factors measured from childhood through middle age: The Muscatine Study. Circulation 2001; 104: 2815-9.

6. Juhola J, Magnussen CG, Viikari JS, Hutri-Kähönen N, Jula A, Lehtimäki T, et al. Tracking of serum lipid levels, blood pressure, and body mass index from childhood to adulthood: the Cardiovascular Risk in Young Finns Study. J Pediatr 2011; 159: 584-90.

7. Webber LS, Srinivasan SR, Wattigney WA, Berenson GS. Tracking of serum lipids and lipoproteins from childhood to adulthood. The Bogalusa Heart Study. Am J Epidemiol 1991; 133: 884-99.

8. Kit BK, Carroll MD, Lacher DA, Sorlie PD, DeJesus JM, Ogden C. Trends in serum lipids among US youths aged 6 to 19 years, 1988-2010. JAMA 2012; 308: 591-600.

9. Dathan-Stumpf A, Vogel M, Hiemisch A, Thiery J, Burkhardt R, Kratzsch J, Kiess W. Pediatric reference data of serum lipids and prevalence of dyslipidemia: Results from a population-based cohort in Germany. Clin Biochem 2016; 49: 740-9.

10. Šimunovic M, Bozic J, Milic L, Unic I, Škrabic V. The Prevalence of Metabolic Syndrome and Cardiovascular Risk Factors in Obese Children and Adolescents in Dalmatia: A Hospital Based Study. Int J Endocrinol 2016 Article ID 1823561, 7 pages.

11. Parray IA, Parry MA, Latief M. Prevalence of dyslipidemia in school children of Kashmir valley. Diabetes Metab Syndr 2016; 10 (2 Suppl 1): S47-54.

12. Zheng $\mathrm{W}$, Chen $\mathrm{Y}$, Zhao A, Xue Y, Zheng Y, Mu Z, Wang P, Zhang Y. Associations of sedentary behavior and physical activity with physical measurements and dyslipidemia in school-age children: a cross-sectional study. BMC Public Health 2016 Nov 24 16(1) 1186.

13. Silva AMBT, Capingana CP, Magalhães $P$, Gonçalves MAA, Molina MB, Rodrigues SL, Baldo MP, Mateus MSB, Mill JG. Cardiovascular risk factors in pre-pubertal schoolchildren in Angola. Cardiovasc J Afr 2016; 27: 315-21.

14. Bibiloni M, Salas R, De la Garza YE, Villarreal JZ, Sureda A, Tur JA. Serum Lipid Profile, Prevalence of Dyslipidaemia, and Associated Risk Factors Among Northern Mexican Adolescents. J Pediatr Gastroenterol Nutr 2016; 63: 544-9.

15. Bianchini de Quadros TM, Pinheiro Gordia A, Rodrigues Silva L, Santos Silva DA, Mota J. Epidemiological survey in schoolchildren: determinants and prevalence of cardiovascular risk factors. Cad Saude Publica 2016 Feb 32(2) e00181514.

16. Barja S, Arnaiz P, Villarroel L, Domínguez A, Castillo O, Farías M, Mardones F. Dislipidemias en escolares chilenos: prevalencia y factores asociados. Nutr Hosp 2015; 31: 2079-87.

17. Bustos P, Sáez K, Gleisner A, Ulloa N, Calvo C, Asenjo S. Metabolic syndrome in obese adolescents. Pediatr Diabetes 2010; 11: 55-60.

18. Cotrim Lima MC, Romaldini CC, Romaldini JH. Frequency of obesity and related risk factors among school children and adolescents in a low-income community. A cross-sectional study. Sao Paulo Med J 2015; 133: 125 30.

19. Lilly CL, Umer A, Cottrell L, Pyles L, Neal W. Examination of cardiovascular risk factors and rurality in Appalachian children. Rural and Remote Health (Internet) 2017; 17: 4200.

20. Carnethon MR, Ayala GX, Bangdiwala SI, Bishop V, Daviglus ML. Association of cardiovascular risk factors between Hispanic/Latino parents and youth: the Hispanic Community Health Study/Study of Latino Youth. Annals of Epidemiology 2017; 27; 260-8.

21. Disponible en: http://web.minsal.cl/portal/url/item/ bcb03d7bc28b64dfe040010165012d23.pdf

22. Disponible en: http://www.ministeriodesarrollosocial. gob.cl/pdf/upload/Informe_de_Desarrollo_Social_2016. pdf

23. Disponible en: http://www.ine.cl/docs/default-source/ FAQ/s\%C3\%ADntesis-de-resultados-censo-2002.pd$\mathrm{f}$ ? $\mathrm{sfvrsn}=2$

24. Disponible en: https://es.scribd.com/document/319272 914/Norma-Para-La-Evaluacion-Nutricional-de-Ninosy-Adolescentes-de-5-a-19-Anos-2016

25. National High Blood Pressure Education Program Working Group on High Blood Pressure in Children 
and Adolescents. The fourth report on the diagnosis, evaluation, and treatment of high blood pressure in children and adolescents. Pediatrics. 2004; 114(2 Suppl 4th Report): 555-76.

26. Friedewald WT, Levy RI, Fredrickson DS. Estimation of the concentration of low-density lipoprotein cholesterol in plasma, without use of the preparative ultracentrifuge. Clin Chem 1972; 18: 499-502.

27. Expert panel on integrated guidelines for cardiovascular health and risk reduction in children and adolescents: summary report. Pediatrics 2011; 128 (Suppl 5): S21356.

28. Vrablík M, Dobiášová $M$, Zlatohlávek L, Urbanová Z, eška R. Biomarkers of cardiometabolic risk in obese/ overweight children: Effect of lifestyle intervention. Physiol Res 2014; 63: 743-52.

29. Barja S, Arnaiz P, Domínguez A, Villarroel L, Cassis B, Castillo $\mathrm{O}$, et al. Normal plasma insulin and HOMA values among Chilean children and adolescents. Rev Med Chile 2011; 139: 1435-43.
30. Disponible en: http://reportescomunales.bcn.cl/2015/ index.php/Carahue

31. Disponible en: http://reportescomunales.bcn.cl/2015/ index.php/Puente_Alto

32. Manninen V, Tenkanen L, Koshinen P, Huttunen JK, Mänttäri M, Heinonen PO, et al. Joint effects of serum triglyceride and LDL colesterol and HDL colesterol concentrations on coronary heart disease in the Helsinsky Heart Study: implications for treatment. Circulation 2002; 85: 37-45.

33. Dobiasova M, Frohlich J. The plasma parameter $\log (\mathrm{TG} /$ HDLC) as an aterogenic index: correlation with lipoprotein particle size and esterification rate in apo B-lipoprotein-depleted plasma (FERDHL). Clin Biochem 2001; 34: 583-8.

34. Nogay NH. Assesment of the correlation between the atherogenic index of plasma and cardiometabolic risk factors in children and adolescents: might it be superior to the TG/HDL ratio? J Pediatr Endocrinol Metab 2017; 30: 947-55. 